\title{
Identification of everyday objects on the basis of fragmented outline versions
}

\author{
Sven Panis, Joeri De Winter, Joachim Vandekerckhove, Johan Wagemans \\ Laboratory of Experimental Psychology, University of Leuven, Tiensestraat 102, B 3000 Leuven, Belgium; \\ e-mail: johan.wagemans@psy.kuleuven.be \\ Received 16 September 2005, in revised form 18 May 2007; published online 22 February 2008
}

\begin{abstract}
Although Attneave (1954 Psychological Review 61 183-193) and Biederman (1987 Psychological Review 94 115-147) have argued that curved contour segments are most important in shape perception, Kennedy and Domander (1985 Perception 14367 -370) showed that fragmented object contours are better identifiable when straight segments are shown. We used the set of line drawings published by Snodgrass and Vanderwart (1980 Journal of Experimental Psychology: Human Learning and Memory 6 174-215), to make outline versions that could be used to investigate this issue with a larger and more heterogeneous stimulus set. Fragments were placed either around the 'salient' points or around the midpoints (points midway between two salient points), creating curved versus relatively straight fragments when the original outline was fragmented (experiment 1), or angular and straight fragments when straight-line versions were fragmented (experiment 2). We manipulated fragment length in each experiment except the last one, in which we presented only selected points (experiment 3). While fragmented versions were on average more identifiable when straight fragments were shown, certain objects were more identifiable when the curved segments or the angles were shown. A tentative explanation of these results is presented in terms of an advantage for straight segments during grouping processes for outlines with high part salience, and an advantage for curved segments during matching processes for outlines with low part salience.
\end{abstract}

\section{Introduction}

Rese archers studying which segments of the contours of an object carry most information for correct identification have drawn contradictory conclusions using different methods. Attneave (1954) suggested that high-curvature regions of an object's contour are more perceptually informative than others. He demonstrated (i) that connecting the high-curvature points of a line drawing of a cat with straight lines does not eliminate recognition, and (ii) that people mark the points with high absolute curvature when asked to mark the 'salient' or important points on a smoothly curved closed contour. Recently, Norman et al (2001) replicated the results of Attneave's second demonstration by having 12 persons copy silhouettes of potatoes - shapes with smoothly varying convex and concave curvatures - using only 10 points, and observing that they consistently marked regions of locally high absolute curvature. With Koenderink and van Doorn (1978), they concluded that sensitivity to curvature maxima is useful for qualitative three-dimensional (3-D) shape perception because curvature maxima are projections of positive or negative Gaussian curvature on the surface of 3-D objects (see also Todd 2004). Similarly, Feldman and Singh (2005) used information theory to show that information along visual contours is concentrated in regions of high magnitude of curvature.

In an unpublished study, Biederman and Blickle (1985) deleted varying amounts of the (internal and external) contours of line drawings of 18 objects $(25 \%, 45 \%$, and $65 \%)$ either at the vertices or at mid-segments. With an exposure duration of $100 \mathrm{~ms}$ and $65 \%$ contour deletion (or 35\% contour shown), they found that removal of vertices resulted in higher error rates than the mid-segment removal, consistent with the studies mentioned above using or assuming closed contours. Biederman's (1987) recognition-by-components 
(RBC) theory, a member of structural decomposition models of shape representation, states that an image is first segmented at deep concavities into an arrangement of volumetric primitives or components (geons) based on non-accidental properties of edges in a 2-D image (curvature, collinearity, symmetry, parallelism, and co-termination). After identification of the primitives, the spatial arrangement between them is matched against stored 3-D structural descriptions. Because vertices are important diagnostic features for the identity of the components, removal of vertices is expected to yield more errors than mid-segment deletion.

However, some theoretical assumptions of Biederman's (1987) theory can be questioned in the light of other findings. First, Kennedy and Domander (1985) used a small number of line drawings of objects and found that identification of fragmented object contours was worse when fragments were place around 'MAX' points (points at which the contour changes direction maximally) than around 'MIN' points (points midway between two MAX points), and best when fragments were occupying positions between MIN and MAX points. Kennedy and Domander (1985, page 369) concluded that "the shapes of objects are best represented by samples of the contour that are selected to be evenly distributed, even if this means eliminating all of the points where contour changes direction maximally", because extrapolation across the gaps between the fragments of the contour is in general more accurate when the gaps are small. According to this extrapolation hypothesis, making fragments longer should never result in a decrease of identification performance. They explain the better performance with MIN fragments compared to MAX fragments of the same physical length by the fact that "in MIN point pictures the distribution of line segments may be superior for showing direction" (Kennedy and Domander 1985, page 369). Second, the results of Cave and Kosslyn (1993) suggest that objects are not necessarily parsed at concavities into their constituent parts prior to recognition, while proper spatial relations between parts are indeed critical for recognition. Third, whereas Biederman and Cooper (1991) interpreted their priming results using fragmented pictures as evidence for an intermediate representation in terms of component parts (see also Hayworth and Biederman 2006), Cave and Kosslyn (1993) argued convincingly that these results can be explained on overall global shape alone (based on completion; see also Edelman 1999). Fourth, competitive grouping of contour segments occurs at all levels of the visual system and the figure-ground assignments are influenced by bottom - up geometrical salience as well as top-down object knowledge (Hess and Field 1999; Murray et al 2004; Palmer et al 2003; Spillmann 1999). Fifth, performance in visual discrimination, classification, and completion tasks seems to be dominated by the configural properties (symmetry, parallelism, etc) between the components (contour segments) regardless of the discriminability of the component properties (length, curvature), suggesting that the configural properties have processing priority and may be available earlier than the component properties (Kimchi 1994; Kimchi and Bloch 1998; Sekuler 1994; Sekuler et al 1994). Finally, Keane et al (2003) showed that configural changes made to object parts were detected more easily and quickly than changes made to the shape of object parts.

These studies all suggest that the global shape, based on the spatial relations between the visible elements of an object, is represented first, while identifying the parts of an object on the basis of concavities (De Winter and Wagemans 2006; Hoffman and Richards 1984; Hoffman and Singh 1997; Singh and Hoffman 2001) occurs later. This is consistent with the reverse-hierarchy theory of vision (Hochstein and Ahissar 2002), with view-based theories which state that configural relations between viewpointdependent object features (not specifically related to parts) are matched directly with stored representations (Lowe 1987; Tarr and Pinker 1989; Ullman 1989), and with recent computational models and theories about visual processing (Rolls and Deco 2002). 
Also, there are a number of reasons to believe that inflections are perceptually important too. Mathematically, a change in curvature sign is easier to compute, more robust, and more stable under transformations than maxima or minima of curvature (Van Gool et al 1994), and inflections on the contour correspond to parabolic lines on the 3-D object surface, separating convex and concave surface regions (Koenderink and van Doorn 1982). Finally, Beintema and Lappe (2002) found that a biologicalmotion stimulus is still interpretable when the lights are attached somewhere to the limbs, instead of the joints. Thus, comparing identification of curved fragments around salient points (positive and negative curvature extrema) with straight fragments around midpoints (often, but not always, close to inflections) is not just interesting for the sake of comparison, but also theoretically meaningful because it can generate testable hypotheses (see section 6).

Here we present a large-scale study, consisting of three experiments, in which we tested Biederman and Blickle's (1985) and Kennedy and Domander's (1985) ideas about which segments of object contours are most informative for object identification, using a large number of objects and a large sample of subjects. This study is part of a larger research program on the role of curvature singularities in shape and object perception (see De Winter and Wagemans 2004 for an overview), for which we created outlines of objects derived from the published set of everyday objects of Snodgrass and Vanderwart (1980; see Wagemans et al 2008). These outlines were used by De Winter and Wagemans (2008a) to test Attneave's (1954) hypothesis more thoroughly. A large number of participants were asked to mark the salient points (SPs) on this large and heterogeneous set of object outlines. Their data confirmed Attneave's hypothesis that salient points marked by subjects are closest to extrema (points with locally a negative or positive extremum of curvature) and even closer to negative minima or concavities than to positive maxima or convexities because in natural shapes concavities are in general deeper than convexities and can thus be located better perceptually.

We use a large number of object outlines $(N=186)$ because previous investigations of picture fragmentation (Biederman and Blickle 1985; Kennedy and Domander 1985) typically used a low number of quite homogeneous object shapes to test their hypotheses. We show that conclusions based on a small number of object stimuli often cannot be generalised to all possible object shapes because the global shape is an important factor in determining whether straight or curved fragments will be important. We use salient points and midpoints instead of extrema and inflections because De Winter and Wagemans (2008b) found that straight-line stimuli based on (subjectively defined) salient points were better identifiable than when based on (mathematically defined) extrema, although, on average, the contours contained more extrema than salient points. In practice, this difference is not so important anyway because De Winter and Wagemans (2008a) have shown that salient points are often very close to strong extrema. The outlines of objects (no internal contours) are used because this (i) avoids differences in occlusion cues (eg vertices) between fragmented versions, (ii) allows us to avoid the presence of internal features that could differ in their diagnosticity for identification between both types of fragmentation used in this study (eg the pedal of a bicycle), and to control (iii) the position and extent of deletions, and (iv) the similarity of the distribution of both sets of resulting fragments more systematically as was done by Biederman and Blickle (1985) and Kennedy and Domander (1985).

For each of our objects we created different types of fragmented versions in which the fragments were evenly distributed. First, when fragmenting the original outlines containing smoothly changing curvature values, we placed the fragments around the salient points (SPs) identified by De Winter and Wagemans (2008a) or around the midpoints (MPs, points on the contour in the middle between two SPs), creating curved 
and more or less straight fragments, respectively. Four different fragment lengths were tested in experiment 1 . Second, in experiment 2, the influence of changing local properties of the image elements (the visible contour segments) was studied by deleting local curvature information from the contour segments by fragmenting the straight-line versions (in which the SPs are connected with straight lines), used by De Winter and Wagemans (2008b). This results in fragments that were either corner-shaped or completely straight. In terms of RBC (Biederman 1987) this deletion of local curvature implies a change in the identity of the components (changing curved into straight edges). Three fragment lengths were tested in experiment 2.

Finally, the necessity of the presence of local direction information around these points for identification was studied by presenting the smallest possible fragments: the SPs and/or the MPs themselves (experiment 3). The main motivation for experiment 3 was that Deregowski (1986) noted that the results of Kennedy and Domander (1985) do not invalidate Attneave's (1954) hypothesis, because he only talks of points at which information is concentrated, while Kennedy and Domander (1985) used short dashes that contained information about direction. However, although Attneave's hypothesis is concerned only with highly informative points, Attneave himself did connect the high-curvature points with straight lines, so he added direction information that was valid in the sense that no spurious angles were introduced. Similarly, when people have to mark salient points, the complete contour is shown. This is the first study in which Deregowski's (1986) strict interpretation of Attneave's hypothesis is tested by presenting high informative points alone.

\section{General methods}

Because all three experiments reported here belong to the same large-scale study, they share several aspects of the methods for data acquisition (subjects, stimuli, procedure) and data analysis (scoring, dependent variables, a posteriori analyses). To avoid repetition in the description of the individual experiments, we include these general aspects of the methods here and focus on the specific details in which the methods differ between experiments below (eg the method of fragmentation).

\subsection{Subjects}

First-year psychology students at the University of Leuven participated in all of the experiments in this study as a mandatory component of their curriculum. They were always naive regarding the purpose of the experiment and unfamiliar with the stimuli (we used different samples with new freshmen in each of three consecutive academic years), and all had normal or corrected-to-normal vision. Depending on the number of conditions in each experiment, we used a different number of subjects, to have data from 18 to 26 subjects per stimulus per condition within a reasonable time per subject (usually less than $20 \mathrm{~min}$ ).

\subsection{Stimuli}

The stimulus set consisted of 17 fragmented versions of object outlines that were derived from the 260 line drawings of everyday objects by Snodgrass and Vanderwart (1980). In a previous study (Wagemans et al 2008), silhouette and outline versions were created of the complete Snodgrass and Vanderwart set (see also De Winter and Wagemans 2004; Wagemans et al 1998). Silhouettes were made by filling-in the interior surfaces of the line drawings in black. Outlines were subsequently extracted automatically and spline-fitted to obtain smooth curvature values at all points along the contour.

Some outlines were excluded for the following reasons. (i) Outlines that were too simple (eg squares or circles) were excluded because of numerous possible valid namings. (ii) Some outlines had some small anomalies in the outline shape (due to the spline-fitting procedure) and they were excluded because these anomalies might affect the fragmented 
versions differentially and hence our major results of interest. These selection criteria led to a set of 186 object outlines (out of 260), with an average identification rate of $82.8 \%(\mathrm{SD}=23.1 \%)$.

In each of the three experiments belonging to the current study, the 186 objects were divided into two groups and subsequently each group was divided into a number of subgroups. We made sure that groups and subgroups were always matched to have approximately the same average identifiability, the same number of living versus nonliving objects, and the same average number of inflections (one of our operationalisations of outline complexity).

\subsection{Procedure}

The experiments were performed in a computer classroom with 33 PCs separated by about $1 \mathrm{~m}$. Each experiment consisted of multiple sessions with a maximum number of 30 subjects per session. We presented all the stimuli centred on a 17-inch CRT display at a viewing distance of approximately $0.7 \mathrm{~m}$, but viewing distance was not strictly controlled. The display resolution was set to $1024 \times 768$ pixels. The refresh rate was $60 \mathrm{~Hz}$. Stimuli were all contained within a box of $640 \times 480$ pixels (not drawn as such), resulting in a viewing angle of about $16 \mathrm{deg} \times 12 \mathrm{deg}$.

Trials were self-paced. Each fragmented version was presented for a maximum of $5 \mathrm{~s}$ and then replaced by a fixation cross. Subjects were asked to identify each stimulus and subsequently input its name via the computer keyboard and click on an $\mathrm{OK}$ button with the mouse when finished. Subjects could begin typing the object name as soon as they had identified the stimulus and they could type and correct as long as they wanted. If the subject clicked on $\mathrm{OK}$ in a time period shorter than $5 \mathrm{~s}$, the stimulus was removed from the screen and the next stimulus appeared. The presentation order was randomised for each subject separately and the experimenter secured silence throughout the session until the last subject was finished.

\subsection{Scoring}

A response was counted as correct when either the same name was given as the one listed by Snodgrass and Vanderwart (1980), or when it was a synonym or colloquial name that clearly indicated the same concept. This was done because we used Flemish subjects in all experiments and Flemish has many more synonyms and colloquial names than English or Dutch (eg Severens et al 2005).

We also approved names referring to related objects if these were not visually distinguishable in our outlines. For example, we approved "aircraft" for "airplane", "cradle" for "baby carriage", "mouth" for "lips", "rat" for "mouse", "nutcracker" for "pliers", etc, but also "dromedary" for "camel" because many people do not know the difference. However, slightly related names that were referring to different basic-level categories were not allowed when they were visually distinguishable in our contour stimuli (eg "seat" for "bed", "bee" for "beetle", "chicken" for "bird", "shoe" for "boot", etc). Scoring was done automatically for all the names that were already contained in our database from previous studies. New names were being scored manually by applying the same criteria (in case of doubt, the authors decided together). The database was updated with the new names (and their scoring) each time a new experiment was performed. In any event, every experiment in this study used the same scoring criteria and the results are therefore comparable across the different experiments.

\subsection{Data analysis}

We determined the average percentage of correct identification in each of the conditions across subjects and compared them with an analysis of variance. Specifically, general linear mixed-model theory (GLMM; Littell et al 1996) was used to model a repeated-measures block design with type of fragment and/or fragment length as 
within-subjects factors and subject as a random block factor. In a GLMM the random subject effects model the correlations between observations of the same subject. We also performed similar analyses across stimuli, but we will not report these results because they are similar to the analyses across subjects.

We also computed a number of stimulus measures on the closed contour to support our tentative interpretations on the basis of stimulus inspection more quantitatively. Correlations between these measures and performance differences (averaged across subjects) between SP and MP versions were overall low (all $r \mathrm{~s}<0.25$ ) but some interesting patterns will be reported. $t$-Tests for independent samples revealed that the two sets of stimuli showing opposite performance patterns across fragment lengths (SP $>$ MP or SP $<$ MP; $N=47$ in each set) differed significantly in (i) the area, (ii) the number of inflections, (iii) the number of peaks which was based on the adaptive smoothing algorithm by Horng (2003), which iteratively smoothes out lowcurvature values and strengthens high-curvature values, resulting in a certain number of peaks in the curvature graph, (iv) a measure of compactness (contour length divided by the square of the area), (v) contour length, (vi) a measure of homogeneity (the number of peaks divided by the square of the contour length), (vii) number of parts (based on the empirical segmentation data of De Winter and Wagemans 2006), (viii) the number of fragments or the number of salient points, (ix) the average of the absolute curvature values of every point of a contour.

\section{Experiment 1: Identification of fragmented outlines}

\subsection{Introduction}

In experiment 1 we fragmented the original, smoothly curved outlines in two ways: fragments were placed around salient points (SPs) or around midpoints (MPs) and four fragment lengths were tested. RBC predicts overall lower identification for MP fragments than for SP fragments.

\subsection{Methods}

\subsubsection{Subjects. 202 first-year, naive psychology students participated.}

3.2.2 Stimuli. De Winter and Wagemans (2008a) asked an independent sample of observers $(N=161)$ to mark the salient points on each contour of the 260 objects of Snodgrass and Vanderwart (1980). The selection of the most salient points proceeded as follows. First, the raw frequency data were smoothed by a Gaussian function (with an SD of 5 pixels) and then the local maxima from this salience distribution were selected if their value was higher than a particular threshold. Because the contours differed widely in how distributed the salience values were, the threshold was set adaptively: it was determined as the integer value of the average smoothed salience (eg 7 for an average smoothed salience of 7.93). It was clear that subjects usually marked points with high curvature.

For the 186 selected object outlines, fragments were placed either around these salient points (SPs) or around midpoints (MPs, the points halfway in-between two SPs with distance measured on the original outline as the Euclidean distance in pixels from point to point). Four fragmentation levels were used: $15 \%, 20 \%, 25 \%$, or $30 \%$ of the total contour was shown (figure 1). We approximated the requested total percentage contour in both conditions by starting from the relevant set of 'target' points (ie SPs or MPs) and letting the fragments grow until each of both parts of a fragment occupied the requested percentage of the distance on the contour between the target point and the neighbouring MPs (in the case of SP targets) or SPs (in the case of MP targets). Thus, each fragment contained an SP or an MP but was not necessarily divided exactly in half by the target point because the distance between the target point and both neighbouring MPs or SPs was not necessarily the same. This procedure results 

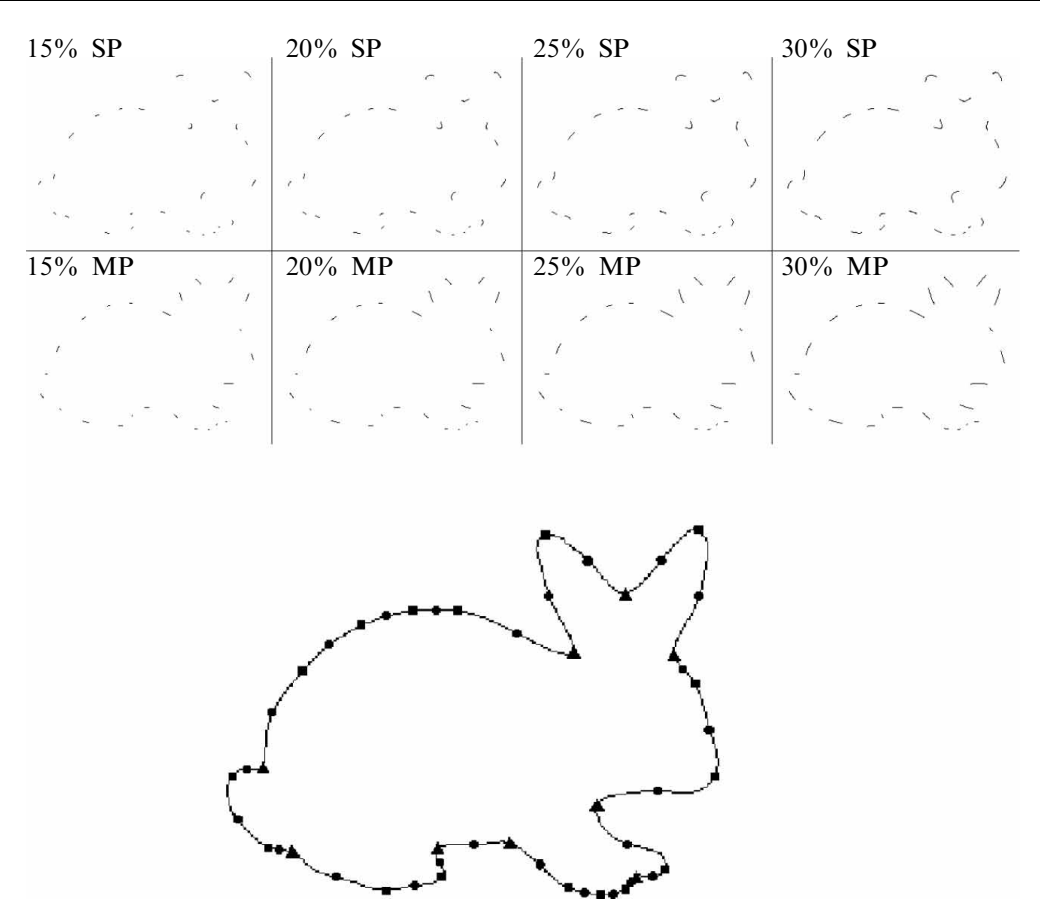

Figure 1. Examples of SP and MP fragments for stimulus No 182 (rabbit) for each fragment length. On the closed contour, triangles indicate salient points with negative curvature, squares salient points with positive curvature, and circles indicate midpoints.

in variable gap and fragment lengths for each object contour. This will contribute to grouping at different levels of the visual system, from simple local filling-in to the global integration of parts in a structural description (Lamote and Wagemans 1999; see also Kourtzi et al 2003 for related neurophysiological evidence). Each fragmented version has the same number of fragments and we will refer to the fragmentation levels by the variable 'fragment length' with values $15 \%, 20 \%, 25 \%$, and $30 \%$. These four fragmentation levels were chosen on the basis of a pilot study where the difference in identification between similar types of mathematically defined fragments (fragments placed around extrema or inflections) was largest in the range $15 \%$ to $28 \%$ (see Wagemans et al 2001).

3.2.3 Procedure. The 186 objects were divided into two groups and each group subsequently into four subgroups containing 23 or 24 objects. Every subject saw each subgroup in one of eight within-subjects conditions ( 2 levels of fragment type $\times 4$ levels of fragment length). Assignment of subgroups to conditions was counterbalanced across subjects. Every participant received 186 trials. Each object was shown in each condition to 25 or 26 subjects.

\subsection{Results and discussion}

First, we calculated the percentage correct responses for each combination of subject $(N=202)$, type of fragment (SP and MP), and fragment length $(15 \%, 20 \%, 25 \%$, and $30 \%$ ). These scores were analysed with a GLMM as a repeated-measures block design with type of fragment and fragment length as within-subjects factors and subject as a random block factor (figure 2 and table 1). Both main effects of fragment type and fragment length were highly significant $\left(F_{1,201}=120.42, p<0.001 ; F_{3,603}=214.65\right.$, $p<0.001)$ : MP fragments were identified correctly more frequently than SP fragments $(62.66 \%$ versus $56.01 \%)$ and correct identification improved with larger fragment 


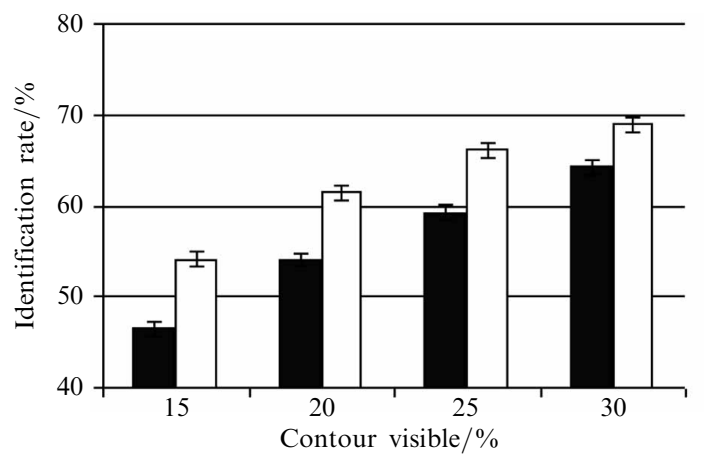

SP

$\square \mathrm{MP}$

Table 1. Descriptive information of the objects in each of three groups (SP $>$ MP, SP $=\mathrm{MP}$, $\mathrm{SP}<\mathrm{MP}$ ), tabulated separately for each experiment and for each fragment length. Each cell contains the number of stimuli, the average percentage identification difference, and the median identification difference. The last column (AV) shows these values when identification performance for each fragment type is first averaged across fragment lengths for each object.

\begin{tabular}{|c|c|c|c|c|c|}
\hline & $15 \%$ & $20 \%$ & $25 \%$ & $30 \%$ & AV \\
\hline \multicolumn{6}{|l|}{ Experiment 1} \\
\hline \multirow[t]{3}{*}{$\mathrm{SP}<\mathrm{MP}$} & 101 & 106 & 99 & 91 & 117 \\
\hline & -23.3 & -20.3 & -20.2 & -18.2 & -15.9 \\
\hline & -19.2 & -15.4 & -15.4 & -16 & -12.9 \\
\hline \multirow[t]{3}{*}{$\mathrm{SP}=\mathrm{MP}$} & 23 & 23 & 37 & 41 & 6 \\
\hline & 0 & 0 & 0 & 0 & 0 \\
\hline & 0 & 0 & 0 & 0 & 0 \\
\hline \multirow[t]{4}{*}{$\mathrm{SP}>\mathrm{MP}$} & 62 & 57 & 50 & 54 & 63 \\
\hline & 14.7 & 13.4 & 14.3 & 14.6 & 9.7 \\
\hline & 12 & 8 & 8 & 8 & 6 \\
\hline & $15 \%$ & $20 \%$ & $25 \%$ & $\mathrm{AV}$ & \\
\hline \multicolumn{6}{|l|}{ Experiment 2} \\
\hline \multirow[t]{3}{*}{ SPSL $<$ MPSL } & 82 & 92 & 70 & 90 & \\
\hline & -17.7 & -15.8 & -19.2 & -13.6 & \\
\hline & -12.1 & -12.6 & -15.4 & -10.5 & \\
\hline \multirow[t]{3}{*}{ SPSL $=$ MPSL } & 21 & 32 & 37 & 8 & \\
\hline & 0 & 0 & 0 & 0 & \\
\hline & 0 & 0 & 0 & 0 & \\
\hline \multirow{3}{*}{ SPSL > MPSL } & 83 & 62 & 79 & 88 & \\
\hline & 13.9 & 15.6 & 13.9 & 10 & \\
\hline & 10.5 & 14.3 & 10.5 & 6.9 & \\
\hline \multicolumn{6}{|l|}{ Experiment 3} \\
\hline \multirow[t]{3}{*}{ SPpt $>$ MPpt } & 81 & & & & \\
\hline & 17.4 & & & & \\
\hline & 11.8 & & & & \\
\hline \multirow{3}{*}{$\mathrm{SPpt}=\mathrm{MPpt}$} & 60 & & & & \\
\hline & 0 & & & & \\
\hline & 0 & & & & \\
\hline \multirow[t]{3}{*}{$\mathrm{SPpt}<\mathrm{MPpt}$} & 45 & & & & \\
\hline & -12.5 & & & & \\
\hline & -10 & & & & \\
\hline
\end{tabular}

Figure 2. Percentage correct identification in experiment 1 as a function of fragment length $(15 \%, 20 \%, 25 \%, 30 \%)$ and fragment type (SP and MP). Error bars indicate standard error of the mean $(\mathrm{SEM}=0.8038)$.

$$
\begin{aligned}
& \text { Experiment } 1 \\
& \mathrm{SP}<\mathrm{MP}
\end{aligned}
$$

$\begin{array}{cc}101 & 106 \\ -23.3 & -20 .\end{array}$

$\begin{array}{ll}-20.2 & -18.2 \\ -15.4 & -16\end{array}$

$-15.9$

$-19.2-15.4$

37

0

41

2.9

0

$\begin{array}{rrrrr}0 & 0 & 0 & 0 & 0 \\ 62 & 57 & 50 & 54 & 63\end{array}$

$\begin{array}{lllll}14.7 & 13.4 & 14.3 & 14.6 & 9.7\end{array}$

$\begin{array}{llllll}12 & 8 & 8 & 8 & 6\end{array}$

xperiment 2

SPSL $<$ MPSL

xperiment 3 
lengths $(50.30 \%, 57.73 \%, 62.68 \%$, and $66.63 \%)$. There was suggestive but inconclusive evidence for an interaction between fragment length and type $\left(F_{3,603}=2.31, p=0.08\right)$. The effect of fragment type was significant at every fragment length $\left(F_{1,737}=61.66 \%\right.$, $56.42 \%, 48.26 \%$, and $22.83 \%$, all $p$ s $<0.001)$, but the difference was numerically larger for smaller fragments.

In line with the results of Kennedy and Domander (1985), we found that object identification on the basis of fragments was easier on average when fragments were placed around MPs than around SPs, for every fragment length tested. However, for each fragment length, there was a considerable range of identification performance across the stimuli. There were relatively more SP versions with low identification rates $(<10 \%)$ than MP versions and more MP versions with high identification rates $(>90 \%)$ than SP versions, but in both versions identifiability was distributed across the whole range from $0 \%$ to $100 \%$ for every length.

Interestingly, identification performance did not necessarily improve with larger fragments. About 51 objects showed at least once a drop of at least $10 \%$ identification when fragments got larger. Thus, the extrapolation hypothesis of Kennedy and Domander (1985) does not hold as generally as they implicitly seem to suggest. In addition to mere 1-D proximity-along-the-contour and collinearity between fragments adjacent on the contour, other configural relations (eg parallelism, symmetry, and convexity relations between segments that are adjacent in the 2-D plane but not along the contour) appear to play a role as well and provide a context which may hamper or facilitate the task at hand (see also Liu et al 1999; Pomerantz et al 1977).

When identification rate was averaged across fragment lengths for each object, about two-thirds of the objects showed better identification in MP than in SP, while one-third showed the opposite pattern (see table 1). To study on which variables both groups of object contours differed, we selected the $25 \%(N=47)$ objects with the strongest difference scores in both groups. $t$-Tests for independent samples (see table 2) indicated significant differences between both groups for area, number of inflections and peaks, compactness and homogeneity, contour length, number of fragments and parts, and the average absolute curvature across all points. Our measures of homogeneity and compactness both have a positive correlation with difference scores for every length (SP\% minus MP\%) meaning that less homogeneous and/or less compact objects tend to be more identifiable with MP fragments than with SP fragments. Area, contour length, and the number of peaks all correlate negatively, meaning that larger objects and/or outlines showing more curvature variation and/or objects with longer contours also benefit from MP fragments. These analyses indicate that more complex outlines

Table 2. Results of $t$-tests for independent samples on the $N=47$ most extreme objects at both ends of the average 'SP - MP' identification distribution in experiment 1 . The average of the outline measures (column 1) of the objects in both groups are shown in columns 2 and 3 . The $t$ - and $p$-values are shown in columns 4 and 5 .

\begin{tabular}{lcrll}
\hline Experiment 1 & SP $<$ MP & SP $>$ MP & $t$ & $p<$ \\
\hline Area & 40179.02 & 29420.77 & 3.37 & 0.01 \\
Number of inflections & 27.49 & 18.81 & 2.84 & 0.01 \\
Peaks & 15 & 9.83 & 2.69 & 0.01 \\
Compactness & 13.53 & 30.21 & 2.56 & 0.02 \\
Length & 1414.51 & 1153.58 & 2.52 & 0.02 \\
Homogeneity & 16.24 & 34.69 & 2.28 & 0.03 \\
AvAbsCurv & 0.29 & 0.2 & 2.2 & 0.04 \\
Number of fragments & 30.19 & 23.4 & 2.17 & 0.04 \\
AvDeltaAbsCurv & 0.04 & 0.02 & 2.13 & 0.04 \\
Number of parts & 5.3 & 4.06 & 2.05 & 0.05 \\
\end{tabular}


with high part salience (less compact, less homogeneous, larger area, longer contour, more parts, etc) benefit from the relatively straight MP fragments, while simpler outlines with low part salience benefit from the curved SP fragments. We will return to these findings in section 6 .

\section{Experiment 2: Identification of fragmented straight-line versions of outlines}

\subsection{Introduction}

We fragmented the straight-line versions connecting SPs used by De Winter and Wagemans (2008b) in two ways to study the effect of deleting smooth changes in local curvature. Three fragment lengths were tested. RBC predicts an overall lower performance than in experiment 1, since the shape of the inferred components (geons) will have changed completely.

\subsection{Methods}

4.2.1 Subjects. 231 first-year, naive psychology students participated.

4.2.2 Stimuli. The same 186 objects as in experiment 1 were used. First, a 'straight-line' (SL) version was created by connecting the neighbouring SPs of every object contour with straight lines (from now on we call these SPs connected by straight-line segments SPSLs). Second, points on this straight-line contour lying in the middle between two SPSLs (the points halfway in-between two SPSLs with distance measured on the straight-line contour as the Euclidean distance in pixels from point to point) were defined as midpoints in the straight-line versions (MPSLs). Last, these straightline contours were fragmented such that the fragments were lying around the SPSLs (forming corner-shaped fragments with variable angles) or the MPSLs (forming short straight lines). Using the same procedure as in experiment 1 , we constructed three fragmentation levels: $15 \%, 20 \%$, or $25 \%$ of the total contour was shown (see figure 3 ). Notice that the SPSLs have the same position as the original SPs, while, in most cases, the MPSLs have a different position than the original MPs. (Note also that the MPSL
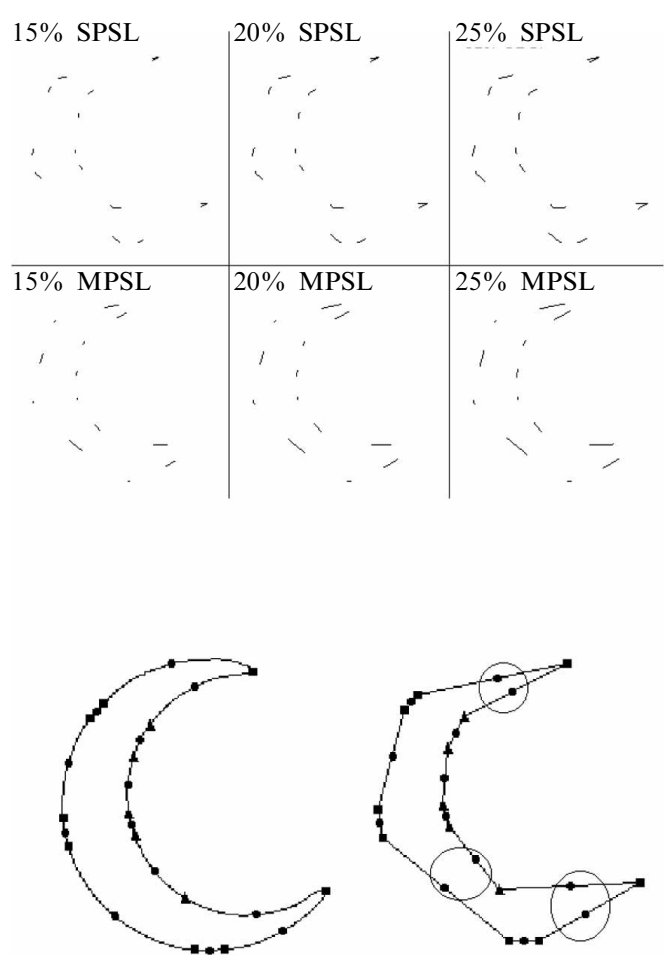

Figure 3. Examples of SPSL and MPSL fragments for stimulus No 146 (moon) for each fragment length. Below, the original and SL contour are shown, with triangles indicating salient points with negative curvature, squares salient points with positive curvature, and circles midpoints. Notice the change in position between the encircled MPSLs and the MPs. 
fragments could have been constructed differently by placing a straight fragment on the tangent at the MPs, so that the MPSLs would also always have the same position as the original MPs.) Furthermore, SPSL fragments adjacent on the contour are by definition perfectly collinear because they derive from straight-line figures while MPSL fragments are not necessarily collinear along the contour. Thus, if we still find an advantage for MPSL fragments compared to SPSL fragments, this means that (i) the absolute position of the straight MPSL fragment is not so important, and (ii) that collinearity is not the only, and possibly not the most important, configural property that is used to identify fragmented object outlines.

4.2.3 Procedure. The 186 stimuli were divided in two groups and each group subsequently into six subgroups, each containing 15 or 16 objects. Each subject was randomly allocated to a group and saw each of the six corresponding subgroups in one of six within-subject conditions ( 2 levels of SL fragment type $\times 3$ levels of fragment length). Assignment of groups to subjects and subgroups to conditions was counterbalanced across subjects. Every participant received 93 trials. Each object was shown in each condition to $18-20$ subjects.

\subsection{Results and discussion}

First, we calculated percentage of correct responses for each combination of subject $(N=231)$, fragment type (SPSL and MPSL), and fragment length $(15 \%, 20 \%$, and $25 \%)$. These scores were analysed as a repeated-measures block design with fragment type and fragment length as within-block factors and subject as a random block factor (figure 4 and table 1). Both main effects were significant $\left(F_{1,230}=9.76, p<0.005 ; F_{2,460}\right.$ $=131.57, p<0.001)$ : MPSL fragments were recognised correctly more frequently than SPSL fragments $(56.80 \%$ versus $54.84 \%)$ and correct identification improved with larger fragment lengths $(50.08 \%, 56.36 \%$, and $61.02 \%)$. The interaction was not significant $\left(F_{2,460}=0.50, p=0.61\right)$. However, the effect of fragment type was only significant with fragment length $20 \%\left(15 \%: F_{1,674}=2.95, p=0.09 ; 20 \%: F_{1,674}=7.59, p<0.01 ; 25 \%\right.$ : $\left.F_{1,674}=2.26, p=0.13\right)$.

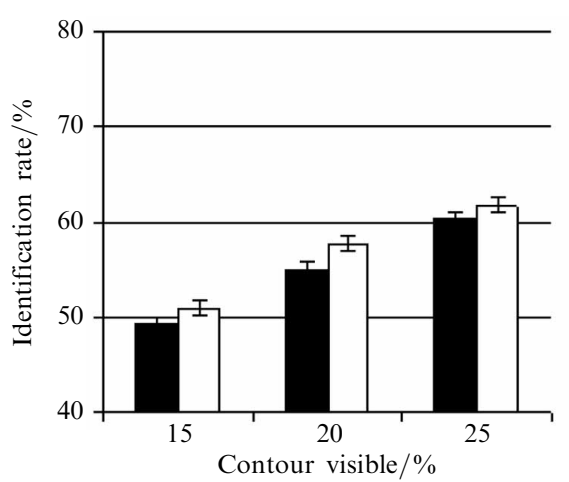

SPSL

$\square$ MPSL

Figure 4. Percentage correct identification in experiment 2 as a function of fragment length $(15 \%, 20 \%$, $25 \%$ ) and fragment type (SPSL and MPSL). Error bars indicate standard error of the mean $(\mathrm{SEM}=0.7662)$.

In spite of the perfect collinearity between SPSL fragments and the orientational and positional differences between MP and MPSL fragments, we still find a better performance with MPSL compared to SPSL, especially for fragment length $20 \%$. However, for each fragment length, there was again a considerable range of identification performance across the stimuli: in both versions identifiability is distributed across the whole range from $0 \%$ to $100 \%$ for every length.

In comparison with experiment 1, identification was higher with SPSL fragments than with SP fragments (probably because of the collinearity between SPSL fragments), but lower with MPSL fragments than with MP fragments (probably because of the positional and orientational differences between MP and MPSL fragments). However, overall, 
performance was not lower than in experiment 1, suggesting that the local curvature in the fragments is not as important as the configural relations between the fragments which delineate the global shape of the outline or the configural relations between view-based convex part-like segments. Also, our measure of homogeneity now has a negative correlation, meaning that more homogeneous objects tend to be more identifiable with MPSL fragments than with SPSL fragments. This is understandable since SPSL fragments will introduce spurious angles for the more homogeneous (ie more circle-like) outlines. When identification rate is averaged across lengths, there are 90 objects that show better identification in MPSL than in SPSL, while 88 objects show the opposite pattern. $t$-Tests for independent samples indicated significant differences between both sets (SPSL > MPSL and SPSL < MPSL; $N=47$ ) only for our measure of homogeneity (average homogeneity for objects showing MPSL > SPSL: 36.6, and for SPSL > MPSL: 16.5, $p<0.02$ ). We will return to these findings in section 6 .

\section{Experiment 3: Identification based on salient points}

\subsection{Introduction}

We tested Deregowski's (1986) strict interpretation of Attneave's hypothesis, ie that the high-curvature points themselves contain most information, by presenting only the SPs, only the MPs, or both. We realise that identification could drop substantially, but even with low identification scores it is interesting to study whether identifiability differs between SPs and MPs. Conceptually, we consider a point to be - in the limitthe smallest possible fragment.

\subsection{Methods}

\subsubsection{Subjects. 124 first-year, naive psychology students participated.}

5.2.2 Stimuli. The same 186 objects as in experiment 1 were used. We presented the SPs alone (SPpt), the MPs alone (MPpt), or all points together (SPMPpt).

5.2.3 Procedure. The 186 stimuli were divided into two groups and each group subsequently into three subgroups, each containing 30 to 32 objects. Each subject was randomly allocated to a group and saw each of the three corresponding subgroups in one of three within-subjects conditions (3 levels of point type). Assignment of groups to subjects and subgroups to conditions was counterbalanced across subjects. Every participant received 93 trials. Each object was shown in each condition to $20-22$ subjects.

\subsection{Results and discussion}

First, we calculated percentage of correct responses for each combination of subject $(N=124)$ and fragment type (SPpt, MPpt, and SPMPpt). These scores were analysed as a repeated-measures block design with fragment type as a within-block factor and subject as a random block factor (see also table 1). The main effect of fragment type was significant $\left(F_{2,246}=501.48, p<0.001\right)$. Tukey-Kramer corrected a posteriori comparisons showed that all three differences are significant. Mean percentage of correct identification in SPpt (17.41) was higher than that in MPpt (12.80), but lower than in SPMPpt (38.85).

We find partial evidence for Deregowski's (1986) strict interpretation of Attneave's hypothesis since, on average, SPs are better identifiable than MPs, but for many objects much information is lost, even when SPs and MPs are presented together. This result shows that a substantial amount of crucial information for identification is present in the orientational information provided by fragments compared to points. Again, there was a considerable range of identification performance across the stimuli: most of the stimuli were hard to identify (102 objects or 55\% have identification rates $<11 \%$ in both the SPpt and MPpt conditions) but in every condition identifiability is distributed across the whole range from $0 \%$ to $100 \%$. 
It is not surprising that, on average, SPs allow better identification than MPs because De Winter and Wagemans (2008b) found that straight-line versions connecting SPs were on average better identifiable than straight lines connecting MPs. Probably people mentally connected the points with virtual lines and this works fine for those objects in which the points that neighbour each other on the contour are closest to each other, or, in other words, in which the density of the points is low enough so that it is clear which points have to be connected. This interpretation is supported by our observation that all objects showing SPpt $>$ MPpt also show SPSL $>$ MPSL. The relative position of the salient points of these objects thus shows the global shape better than for other objects.

\section{General discussion}

It is not surprising that cartoonists need to draw only a few lines to evoke object recognition when our visual system is highly sensitive to view-based configural relations between contour segments in fragmented object contours. Here we tested the identifiability of different fragmented object outlines. In experiment 1 we observed that the relatively straight MP fragments from the original bounding contour are on average more identifiable than SP fragments for every fragment length tested, consistent with the findings of Kennedy and Domander (1985). In experiment 2 we fragmented straight-line versions connecting SPs and we found that the straight MPSL fragments are more easily identified than the angular SPSL fragments (especially for $20 \%$ contour), in spite of the perfect collinearity between SPSL fragments and the possible changes in position and orientation of MPSL fragments compared to the MP fragments from the original outline. In contrast, when points were presented in experiment 3, SPs led on average to higher identification rates than MPs. Since identifiability was lowest in experiment 3, we find only partial evidence for Deregowski's (1986) strict interpretation of Attneave's (1954) hypothesis that most information is concentrated in the salient points themselves.

However, the generality of these findings has to be questioned because (i) in every experiment and for every fragment length tested, our manipulations affected different objects to different extents, (ii) in every experiment and for every fragment length tested, a considerable number of objects showed a pattern opposite to the average pattern (see table 1), (iii) objects showing SP $>$ MP are not necessarily showing SLSP $>$ SLMP or vice versa, and finally (iv) taking the first two experiments together, some objects were identified $>85 \%$ correct in every experimental manipulation $(N=16)$, while others were almost never identified correctly $(<15 \%, N=6)$. The latter finding suggests that diagnostic identity information can be present at different structural levels, eg from the global shape (the configural relations between convex part-like segments) to more local and detailed levels (eg the exact position and shape of a curved fragment, or the exact configural relation between two fragments). So, on top of the average differences between conditions, there is a large variability as well.

One interpretation of this variability is that the visual system is very sensitive to the configural properties between the fragments, and that the configural relations change with changing fragment properties. For example, when local curvature information is deleted by presenting SPSL and MPSL fragments, performance patterns can change dramatically compared to SP and MP fragments (as shown by the fact that SPSL - MPSL difference scores do not correlate with compactness, area, length, and number of peaks as do the SP - MP difference scores, and by the fact that homogeneity correlates positively with SP - MP scores, but negatively with SPSL - MPSL scores). Also, when fragments get larger, there can be a large change in whether or not some configural properties still exist or emerge between two fragments. 
This observed sensitivity to configural properties is consistent with the observed dominance of global, configural properties between fragments during completion (Kimchi 1994; Kimchi and Bloch 1998; Sekuler 1994; Sekuler et al 1994) and suggests that the global shape of objects is an important factor for determining which fragments of the contour are useful for identification. Indeed, psychophysical, anatomical, and neurophysiological research on contour integration has shown that the local orientationspecific interactions between neurons in early visual cortex are context dependent, and are involved in perceiving closure and figure-ground assignment (see Kovács 1996 for a review). Again, such context-dependence is consistent with our findings that MP fragments tend to convey more identification information than SP fragments for complex object outlines (less homogeneous, less compact, more peaks, more inflections, higher average absolute curvature, a longer contour, a larger area, more fragments or salient points, and more parts), while curved SP fragments convey more information for outlines with the opposite characteristics (see table 2). Since we defined outline homogeneity as the number of strong extrema divided by contour length squared, this measure will increase when there are more 'strong' extrema and/or when the contour gets shorter. Because the contours are closed, higher outline homogeneity values (eg when the contour gets shorter for a constant number of strong extrema) will indicate lower part salience and vice versa. Because part salience (see De Winter and Wagemans 2008a; Hoffman and Singh 1997) is defined by the combination of these measures (compactness, outline complexity, contour length, area, number of strong extrema), our results suggest that complex outlines with high part salience show an MP advantage, while simple outlines with low part salience show an SP advantage.

An influence of global factors has also been observed in other studies with the same stimulus set; for example, De Winter and Wagemans (2008b, 2006) found global influences on identifiability of straight-line versions and on segmentation, respectively. To study the influence of global outline characteristics on identifiability of fragmented versions a posteriori, we grouped the objects according to their performance patterns (SP $>$ MP or $\mathrm{SP}<\mathrm{MP}$, and/or SPSL $>$ MPSL or SPSL $<$ MPSL). This revealed some interesting observations. For example, objects showing $\mathrm{SP}<\mathrm{MP}$ but SPSL $>$ MPSL are mostly man-made and have a global shape with many straight segments and angles, so that the perfect alignment between SPSLs is resembling the true shape much better than is the case with SPs. In contrast, objects that show SP $>$ MP, but SPSL $<$ MPSL, have in general contours that are curved and consist of few parts. These objects require local curvature information to be well identifiable and suffer from the presence of sharp corners in SPSLs. Also, highly identifiable objects $(74 \%-100 \%$ in each of 14 fragment conditions) showing SP = MP and SPSL = MPSL have relatively complex contours and a characteristic global shape without much diagnostic information at the level of small details (many animals and symmetrical objects). In contrast, objects which have an MP advantage (SP $<$ MP and SPSL $<$ MPSL) have many parts and long complex contours. Some of these objects suffer from cluttering or grouping ambiguity in the SP and SPSL conditions, while for other objects, some MP and MPSL fragments contain diagnostic configural information that is not explicitly present in the SP and SPSL fragments. Objects which have an SP advantage (SP > MP and SPSL > MPSL) are very compact and homogeneous and contain a critical curved feature that is not explicitly present in the MP and MPSL conditions. Also, objects showing SP = MP and SPSL < MPSL have mainly curved segments in their contour, and most of these objects have few parts and are symmetrical. Some even show better identification with SP fragments than with the complete SP figure (reported by De Winter and Wagemans 2008b), because their global shape is smoothly curved and therefore the SP figure resembles the original shape less than the SP fragments for which the completion process can fill-in curved segments. These observations 
thus reveal the same general trend as was found in De Winter and Wagemans (2008b), ie all fragment versions of sufficiently complex object contours without distinctive features at the level of small details can generally be identified well on the basis of the global shape, regardless of the type of fragment (salient point versus midpoint). Conversely, shapes that are hard to identify from the complete contour $(<20 \%)$ can never be identified from fragments. When other object contours are fragmented, it is the specific selection of fragments that will determine whether identification is still possible: when the grouping process preserves the global shape characteristics or more local distinctive configural features, identification is good and otherwise it is poor.

To understand why the global shape determines whether straight or curved fragments enjoy an identification advantage, we need to understand the effect of shape complexity on grouping and matching processes. Because complex shapes are structurally dissimilar, while more simple shapes are structurally similar (Donderi 2006), and because Gerlach et al $(2004,2006)$ suggested that high structural similarity is an advantage during grouping, but not during matching, we propose the following tentative explanation for our results. First, because fragmenting object outlines that are complex and have high part salience will lead to difficult grouping of the fragments and, once grouped, to easy matching because of their structural variability or outline diagnosticity, we hypothesise that complex outlines will enjoy an MP advantage during grouping processes because the configural relations (symmetry, collinearity, proximity, etc) are stronger between straight fragments than between curved fragments of the same length. This explanation is consistent with the results of Singh and Fulvio (2005), who studied extrapolation of contour geometry and found (i) that extrapolation of curvature increases linearly with the curvature of the inducing contour, and (ii) that the overall precision of the extrapolated contour decreases systematically with curvature. Second, because fragmenting simple outlines with low part salience will lead to easy grouping but difficult matching, we hypothesise that simple outlines will enjoy an SP advantage during matching, because many object representations will be activated that are structurally similar to the input object. Testing these alternatives by comparing stored information with the input can be done better with curved SP fragments because only they contain exact information about the location and shape of part boundaries (concavities) and part end-points (convexities).

Dissociating between competitive grouping between configural properties and matching processes can also explain why information theory and perceptual salience studies indicate extrema as important points in closed shapes, while some identification studies (Kennedy and Domander 1985; but see Biederman and Blickle 1985) found that fragments around midpoints are more identifiable than fragments around extrema. In particular, when points are presented alone, the only information conveyed is relative (and absolute) position. We have shown in experiment 3 that much information is lost when only certain points of the outline are shown (only 27 objects show at least $50 \%$ identification in SPpt or MPpt) indicating a grouping problem. In other words, it seems invalid to reason in terms of information concentration in points, since shape depends on the configural relations between points and fragments. The fact that none of our stimulus measures correlated significantly with the SPpt - MPpt difference suggests that other factors related to 'clutter' determine whether identification based on points is possible, for example, the difference between the distance between points along the contour and the distance between points in the 2-D plane.

However, when small fragments are added, orientation and curvature information is available and configural properties such as collinearity (Claessens and Wagemans 2005), symmetry (Wagemans 1995, 1997), parallelism, etc, can come into play. The results of experiment 1 showed that these configural properties are very powerful: many objects ( $N=46$ or $25 \%$ for SP fragments, and $N=65$ or $35 \%$ for MP fragments) 
are identifiable (by at least $80 \%$ of our subjects) when only $15 \%$ of the contour is shown (called MP-15 and SP-15). Furthermore, we found that MP fragments add more information to MPs (from $12.8 \%$ for MPpt to $54.3 \%$ for MP-15) than SP fragments to SPs (from $17.4 \%$ for SPpt to $46.5 \%$ for SP-15), consistent with an MP advantage during difficult grouping conditions (small fragments and/or complex shapes). It could therefore be interesting to study whether configural properties other than collinearity are detected more easily between straight than between curved segments. Although Kimchi (1994) did not test this hypothesis explicitly, there is a trend in her data which is consistent with this idea. However, we hypothesise that when the configural grouping of the fragments will extract a global shape that is simple and which will activate many candidate objects, then matching processes will benefit from the SP fragments because they contain exact information about location and shape of part boundaries and part end-points.

Finally, when large fragments are added so that closure is possible, or when the complete contour is shown, the grouping process always generates a valid solution and there is no competition between configural relations. Because subjects will be matching the closed contours to memory, the areas that are perceptually salient parts will be the extreme curvature areas. We hypothesise that the presentation of closed contours is the simple reason why salience studies find that extrema are more important for shape representation. Similarly, Feldman and Singh (2005) started from a nonfragmented line when they applied information theory to show that curved segments contain more information compared to straight segments, thereby 'bypassing' any effect of competitive grouping processes.

Theories such as RBC (Biederman 1987) that require that the parts are identified on the basis of detecting concavities, before the object can be identified, would not predict the following observations. First, the deletion of local curvature in experiment 2 did not result in an overall lowering of the performance and affected only a subset of all objects to different (positive and negative) extents. Second, we noticed that some stimuli were identified differently depending on the perceived facing (left or right). For example, a right-facing snail was sometimes identified as a left-facing whale. Pavlova et al (2002) noticed a similar top-down influence of apparent direction of locomotion on the perceptual interpretations of biological-motion stimuli presented normally or backwards. It seems that the initial global interpretation of the facing of the fragmented stimulus influences the perceptual interpretation and the perceived local groupings of the fragments, and inhibits the intended groupings (see also Bruner and Potter 1964). Third, Feldman and Singh (2005) used information theory to show that concavities contain more information than convexities, but only under the assumption of closure, ie only when grouping processes have extracted the global shape. Logically this implies that discriminating between convexities and concavities is possible only when the global shape is represented. Indeed, Barenholtz and Feldman (2003) showed that the single-part superiority effect-faster perceptual comparisons when crossing curvature maxima (convexities) than minima (concavities) - disappeared when the global configuration was not consistent with a part-boundary interpretation (see also Vandekerckhove et al 2007). Finally, initial evidence consistent with the hypotheses of an early MP grouping advantage for complex outlines, and a later SP matching advantage for simple outlines, is provided by Panis and Wagemans (2007). Their results suggest that the initial contact with memory is based on the global shape that is extracted early by configural grouping processes operating in parallel, and not on the detection of concavities and discriminating them from convexities in parallel. We thus conclude that the contradictory results of Biederman and Blickle (1985) and Kennedy and Domander (1985) are mainly due to (i) their unrepresentative choice of shapes 
with too little variation in outline complexity, and partly to (ii) their unsystematic control of the distribution of fragments and extent of deletions.

To summarise, we have shown that the salient segments in closed outlines are not necessarily the most informative or perceptually relevant when fragmented outlines have to be identified. Which fragments contain the most information is influenced by the configural properties between the fragments, and therefore also by the fragment properties (curvature, length, absolute position) and by the complexity of the inferred global shape. Our tentative explanation for the interaction between part salience or outline complexity and fragment curvature is that straight fragments enjoy an identification advantage for complex shapes when grouping is difficult but matching easy, while curved fragments enjoy an identification advantage for simple shapes when grouping is easy but matching difficult. Compared to the introspection in salience studies where the complete shape is represented before points are selected, we think that systematically controlled fragmentation is a good operationalisation to find the most informative regions for object identification because only minimal information is shown.

Acknowledgments. This research was supported by a research grant from the University of Leuven Research Council (OT/00/007) and from the Research Foundation (FWO Flanders, G.0189.02N). This study is part of a larger research program with financial support from the University Research Council (GOA/2005/03-TBA) to the Laboratory of Experimental Psychology. We want to thank two anonymous reviewers for their helpful comments on an earlier version of this article.

\section{References}

Attneave F, 1954 "Some informational aspects of visual perception" Psychological Review 61 $183-193$

Barenholtz E, Feldman J, 2003 "Visual comparisons within and between object parts: Evidence for a single-part superiority effect" Vision Research 431655 - 1666

Beintema J A, Lappe M, 2002 "Perception of biological motion without local image motion" Proceedings of the National Academy of Sciences of the USA $995661-5663$

Biederman I, 1987 "Recognition-by-components: A theory of human image understanding" Psychological Review 94115 - 147

Biederman I, Blickle T, 1985, referenced and discussed in Biederman 1987

Biederman I, Cooper E E, 1991 "Priming contour-deleted images: Evidence for intermediate representations in visual object recognition" Cognitive Psychology 23 393-419

Bruner J S, Potter M C, 1964 "Interference in visual recognition" Science 144 424-425

Cave C B, Kosslyn S M, 1993 "The role of parts and spatial relations in object identification" Perception $22229-248$

Claessens P M, Wagemans J, 2005 "Perceptual grouping in Gabor lattices: proximity and alignment" Perception \& Psychophysics $671446-1459$

Deregowski J B, 1986 "Shape and contour: Dots and dashes" Perception 15217

De Winter J, Wagemans J, 2004 "Contour-based object identification and segmentation: Stimuli, norms and data, and software tools" Behavior Research Methods, Instruments and Computers 36 604-624

De Winter J, Wagemans J, 2006 "Segmentation of object outlines into parts: A large-scale integrative study" Cognition $99275-325$

De Winter J, Wagemans J, 2008a "Perceptual saliency of points along the contour of everyday objects: A large-scale study" Perception \& Psychophysics 70 50-64

De Winter J, Wagemans J, 2008b "The awakening of Attneave's sleeping cat: Identification of everyday objects on the basis of straight-line versions of outlines" Perception $37245-270$

Donderi D C, 2006 "Visual complexity: A review" Psychological Bulletin 132 73-97

Edelman S, 1999 Representation and Recognition in Vision (Cambridge, MA: MIT Press)

Feldman J, Singh M, 2005 "Information along contours and object boundaries" Psychological Review $112243-252$

Gerlach C, Law I, Paulson O B, 2004 "Structural similarity and category-specificity: A refined account" Neuropsychologia $421543-1553$

Gerlach C, Law I, Paulson O B, 2006 "Shape configuration and category-specificity" Neuropsychologia $441247-1260$ 
Hayworth K J, Biederman I, 2006 "Neural evidence for intermediate representations in object recognition" Vision Research $464024-4031$

Hess R, Field D, 1999 "Integration of contours: New insights" Trends in Cognitive Sciences 3 $480-486$

Hochstein S, Ahissar M, 2002 "View from the top: Hierarchies and reverse hierarchies in the visual system" Neuron $36791-804$

Hoffman D D, Richards W A, 1984 "Parts of recognition" Cognition 18 65-96

Hoffman D D, Singh M, 1997 "Salience of visual parts" Cognition 63 29-78

Horng J-H, 2003 "An adaptive smoothing approach for fitting digital planar curves with line segments and circular arcs" Pattern Recognition Letters $24565-577$

Keane S K, Hayward W G, Burke D, 2003 "Detection of three types of change to novel objects" Visual Cognition $10101-127$

Kennedy J M, Domander R, 1985 "Shape and contour: The points of maximum change are least useful for recognition" Perception 14 367-370

Kimchi R, 1994 "The role of wholistic/configural properties versus global properties in visual form perception" Perception 23 489-504

Kimchi R, Bloch B, 1998 "Dominance of configural properties in visual form perception" Psychonomic Bulletin and Review $5135-139$

Koenderink J J, Doorn A J van, 1978 "How an ambulant observer can construct a model of the environment from the geometrical structure of the visual inflow", in Kybernetic Eds G Hauske, E Butenandt (1997, Munich: Oldenbourg) pp 224-247

Koenderink J J, Doorn A J van, 1982 "The shape of smooth objects and the way contours end" Perception $11129-137$

Kourtzi Z, Tolias A S, Altmann C F, Augath M, Logothetis N K, 2003 "Integration of local features into global shapes: Monkey and human fMRI studies" Neuron $37333-346$

Kovács I, 1996 "Gestalten of today: early processing of visual contours and surfaces" Behavioural Brain Research $821-11$

Lamote C, Wagemans J, 1999 "Rapid integration of contour fragments: From simple filling-in to parts-based shape description" Visual Cognition 6345 -361

Littell R C, Milliken G A, Stroup W W, 1996 SAS System for Mixed Models (SAS Institute Leuven. ISBN/ISSN: 1-55544-779-1)

Liu Z, Jacobs D W, Basri R, 1999 "The role of convexity in perceptual completion: beyond good continuation" Vision Research $394244-4257$

Lowe D G, 1987 "Three-dimensional object recognition from single two-dimensional images" Artificial Intelligence $\mathbf{3 1} 355-395$

Murray S O, Schrater P, Kersten D, 2004 "Perceptual grouping and the interactions between visual cortical areas" Neural Networks 17 695-705

Norman J F, Phillips F, Ross H E, 2001 "Information concentration along the boundary contours of naturally shaped solid objects" Perception 30 1285-1294

Palmer S E, Brooks J L, Nelson R, 2003 “When does grouping happen?" Acta Psychologica 114 $311-330$

Panis S, Wagemans J, 2008 "Time-course contingencies in perceptual organization and identification of fragmented object outlines" Journal of Experimental Psychology: Human Perception and Performance accepted

Pavlova M, Krägeloh-Mann I, Birbaumer N, Sokolov A, 2002 "Biological motion shown backwards: the apparent-facing effect" Perception 31435 - 443

Pomerantz J R, Sager L C, Stoever R J, 1977 "Perception of wholes and of their component parts: Some configural superiority effects" Journal of Experimental Psychology: Human Perception and Performance $3422-435$

Rolls E T, Deco G, 2002 Computational Neuroscience of Vision (Oxford: Oxford University Press)

Sekuler A B, 1994 "Local and global minima in visual completion: effects of symmetry and orientation" Perception $23529-545$

Sekuler A B, Palmer S E, Flynn C, 1994 "Local and global processes in visual completion" Psychological Science $5260-267$

Severens E, Van Lommel S, Ratinckx E, Hartsuiker R J, 2005 "Timed picture naming norms for 590 pictures in Dutch" Acta Psychologica 119159 - 187

Singh M, Fulvio J M, 2005 "Visual extrapolation of contour geometry" Proceedings of the National Academy of Sciences of the USA $102939-944$

Singh M, Hoffman D D, 2001"Part-based representations of visual shape and implications for visual cognition", in From Fragments to Objects: Grouping and Segmentation in Vision Eds T F Shipley, P J Kellman (Amsterdam: Elsevier) pp 401 - 459 
Snodgrass J G, Vanderwart M, 1980 "A standardized set of 260 pictures: Norms for name agreement, image agreement, familiarity, and visual complexity" Journal of Experimental Psychology: Human Learning and Memory $6174-215$

Spillmann L, 1999 "From elements to perception: Local and global processing in visual neurons" Perception 281461 - 1492

Tarr M J, Pinker S, 1989 "Mental rotation and orientation-dependence in shape recognition" Cognitive Psychology $21233-282$

Todd J T, 2004 "The visual perception of 3D shape" Trends in Cognitive Sciences 8 115-121

Ullman S, 1989 "Aligning pictorial descriptions: An approach to object recognition" Cognition 32 $193-254$

Vandekerckhove J, Panis S, Wagemans J, 2007 "The concavity effect is a compound of local and global effects" Perception \& Psychophysics 691253 - 1260

Van Gool L, Moons T, Pauwels E, Wagemans J, 1994 "Invariance from the Euclidean geometer's perspective" Perception $23547-561$

Wagemans J, 1995 "Detection of visual asymmetries" Spatial Vision 9 9-32

Wagemans J, 1997 "Characteristics and models of human symmetry detection" Trends in Cognitive Sciences $1346-352$

Wagemans J, De Winter J, Op de Beeck H, Ploeger A, Beckers T, Vanroose P, 2008 "Identification of everyday objects on the basis of silhouette and outline versions" Perception 37 207-244

Wagemans J, Notebaert W, Boucart M, 1998 "Lorazepam but not diazepam impairs identification of pictures on the basis of specific contour fragments" Psychopharmacology 138 326-333

Wagemans J, Panis S, De Winter J, Op de Beeck H, 2001 "Perceptual and conceptual priming in picture identification on the basis of contour fragments with specific curvature properties" Journal of Vision 197 a (abstract of paper presented at VSS, Sarasota, USA) 


\section{PERTEPTION}

VOLUME 372008

www.perceptionweb.com

Conditions of use. This article may be downloaded from the Perception website for personal research by members of subscribing organisations. Authors are entitled to distribute their own article (in printed form or by e-mail) to up to 50 people. This PDF may not be placed on any website (or other online distribution system) without permission of the publisher. 